\title{
Editorial
}

\section{Self-Assembly Techniques for Nanofabrication}

\author{
Amir Kajbafvala, ${ }^{1}$ Hamed Bahmanpour, ${ }^{2}$ Mohammad H. Maneshian, ${ }^{3}$ and Minghang $\mathrm{Li}^{3}$ \\ ${ }^{1}$ Department of Materials Science and Engineering, North Carolina State University, 911 Partners Way, \\ Engineering Building I, Raleigh, NC 27695-7907, USA \\ ${ }^{2}$ Department of Chemical Engineering and Materials Science, University of California, Davis, CA 95616, USA \\ ${ }^{3}$ Department of Materials Science and Engineering, University of North Texas, Denton, TX 76203, USA
}

Correspondence should be addressed to Amir Kajbafvala; akajbaf@ncsu.edu

Received 8 October 2013; Accepted 8 October 2013

Copyright ( $\odot 2013$ Amir Kajbafvala et al. This is an open access article distributed under the Creative Commons Attribution License, which permits unrestricted use, distribution, and reproduction in any medium, provided the original work is properly cited.

Self-assembly is a type of process in which a disordered system with preexisting components forms an organized structure or pattern as a consequence of specific, local interactions among its components without any external direction. Self-assembly of nanoparticles and subsequent formation of well-defined micro- and nanostructures have multiple interesting applications in nanosciences and nanotechnology. Such a mechanism relies on weak noncovalent bonds, such as hydrogen and ionic bonds, or van der Waals and hydrophobic interactions, and it can be applied to fabricate various complex micro-and nanostructures, such as flowers, tubes, rods, micelles, films, membranes, mesophases, particles, and hollow sphere structures.

This special issue addresses the research studies on fabrication, processing, and applications of self-assembled nanostructures. It contains sixteen articles including two reviews and fourteen research articles.

In their paper "A three-dimensional enormous surface area aluminum microneedle array with nanoporous structure," $\mathrm{P}$. C. Chen et al. presented a convenient and cheap method to fabricate a large surface area 3D microneedle array by micromachining, electrolyte polishing, and anodizing processes. The aluminum microneedle array can be used for chemical or biological detection. It can also be applied to drug delivery or storage applications because it can approach painless injection/extraction and high capacity. Their results provide a concept that the microneedle covered with nanopore films such as $\mathrm{TiO}_{2}, \mathrm{WO}_{3}, \mathrm{Ta}_{2} \mathrm{O}_{5}$, or $\mathrm{MoO}_{3}$ can be formed by micromachining, electropolishing, and anodization processes.
In "Synthesis and self-assembly of gold nanoparticles by chemically modified polyol methods under experimental control," N. V. Long et al. studied surface self-attachment and self-assembly of the as-prepared Au nanoparticles during evaporation of the mixture of ethanol and Au nanoparticles without the use of any additives as well as biomolecule linkers. They showed that the specific collective interactions of the nanoparticles with liquid and evaporation of liquid can be controlled by the self-assembly of the arrangements of $\mathrm{Au}$ nanoparticles. The absorption spectra of the solutions of ethanol and the as-prepared Au nanoparticles show the strong SPR phenomenon.

In their paper "The geometry variation of as-grown carbon coils with Ni layer thickness and hydrogen plasma pretreatment," Y. C. Jeon et al. synthesized carbon coils using $\mathrm{C}_{2} \mathrm{H}_{2} / \mathrm{H}_{2}$ as source gases and SF6 as an incorporated additive gas under thermal chemical vapor deposition system. Ni layer on $\mathrm{SiO}_{2}$ substrate was used as a catalyst for the formation of carbon coils. Ni powder was evaporated to form Ni layer on the substrate. The formation densities, morphologies, and geometries of as-grown carbon coils on the substrate were investigated as a function of the evaporation time for Ni catalyst layer formation. The characteristics of as-grown carbon coils with or without hydrogen plasma pretreatment process were also investigated.

In their review "Devastated crops: multifunctional efficacy for the production of nanoparticles," G. Madhumitha and S. M. Roopan discussed that integration of green chemistry principles to nanotechnology is one of the key issues in nanoscience 
research. Biological methods were used to synthesize metal and metal oxide nanoparticles of specific shape and size since they enhance the properties of nanoparticles in greener route. Plant mediated methods devoid the use of toxic chemicals in the synthetic protocols which has adverse effects on the environment.

In their paper "Influence of anode area and electrode gap on the morphology of $\mathrm{TiO}_{2}$ nanotubes arrays," M. Wang et al. proposed that the surface morphology of $\mathrm{TiO}_{2}$ nanotube arrays which were used in the photocatalytic degradation of total volatile organic compounds can be modified by anodization of $\mathrm{Ti}$ foils in EG solution consisting of $0.4 \%$ $\mathrm{NH}_{4} \mathrm{~F}$ and $5 \mathrm{wt} \% \mathrm{H}_{2} \mathrm{O}$ using the appropriate anode area and electrode gap. The effect of anode area and electrode gap on surface morphology of $\mathrm{TiO}_{2}$ nanotube arrays was investigated, and the results show that the highly ordered $\mathrm{TiO}_{2}$ nanotube arrays cannot be formed with inappropriate anode area and electrode gap.

In their review "Function of nanocatalyst in chemistry of organic compounds revolution: an overview," K. Hemalatha et al. performed a literature survey on the subject of nanomaterials application in organic synthesis. The use of green nanocatalyst for the synthesis of various heterocycles has advantages such as short reaction time, high yield, inexpensive chemicals usage, easy work-up procedure, and very specific reaction. In most of the reactions, the spent catalyst can be easily separated from the reaction mixture, also it can be reused without noticeable change in its catalytic activity. A wide range of original procedures for synthesizing various classes of organic compounds, including organic functional group transformation, have been developed on the basis of nanoparticles.

In their paper "A covalently imprinted photonic crystal for glucose sensing," F. Xue et al. produced a glucose-responsive photonic crystal hydrogel by synthesizing hydrogel in the void of PMMA photonic crystal template followed by removing the template. Molecular imprinting improved the selectivity of the photonic crystal-responsive hydrogel towards glucose. Improvement of the current CIPC technology aims to optimize the CIPC composition to make the diffraction visible, thus allowing promising clinical application in noninvasive, real-time glucose monitoring.

In "Solvent-dependent self-assembly of 4,7-dibromo-5,6bis(octyloxy)benzo[c] [1,2,5] thiadiazole on graphite surface by scanning tunneling microscopy," B. Zha et al. investigated selfassembly of DBT molecule on HOPG surface in different solvents by STM. Dramatic differences in the $2 \mathrm{D}$ ordering are observed depending on the nature of solvents. When using 1phenyloctane, a linear structure is fabricated. Br-Br halogen bonds are found and were responsible for the formation of the packing pattern. However, a lamellar structure is formed by using 1-octanoic acid or 1-octanol as the solvent, in which the solvent molecules act as a coadsorbed component due to the hydrogen bonding between DBT molecules and solvent molecules rather than a dispersant.

In, "QDs supported on langmuir-blodgett films of polymers and gemini surfactant", T. Alejo et al. used different Langmuir-Blodgett (LB) films of poly (octadecene-co-maleic anhydride), PMAO, PS-MA-BEE, and Gemini surfactant ethyl-bis to study the effect of the substrate coating on the surface self-assembly of CdSe quantum dots (QDs). Results showed that all the "coating molecules" avoid the 3D aggregation of QDs observed when these nanoparticles are directly deposited on mica. Different morphologies were observed depending on the molecules used as coatings, and this was related to the surface properties, such as wetting ability and the morphology of the coated LB films.

In "Self-assembly of ternary particles for tough colloidal crystals with vivid structure colors," B. Bao et al. proposed that large-scale, robust, intense, and structurally color-tunable polymer crystal films can be fabricated by casting method. This method is not only simple and feasible, but the asobtained polymer crystal films also display brilliant visual iridescence under natural lighting conditions. Under the optimal conditions, the obtained material has the best quality with suitable film forming rate, which provides basis for the mass production and application of the colloidal crystal films with carbon black dopant in coating, cosmetics, and pigments.

In "Self-assembly and soft material preparation of binary organogels via aminobenzimidazole/benzothiazole and acids with different alkyl substituent chains," T. Jiao et al. studied the gelation behaviors of binary organogels composed of aminobenzimidazole/benzothiazole derivatives and benzoic acid with single/multialkyl substituent chain in various organic solvents. Results showed that the number and length of alkyl substituent chains, and benzimidazole/benzothiazole segment, have played a crucial role in the gelation behavior of all gelator mixtures in various organic solvents. The length of alkyl substituent chains has also played an important role in changing the gelation behaviors and assembly states. Morphological studies revealed that the gelator molecules selfassembled into different aggregates from wrinkle, lamella, and belt to fiber with change of solvents.

In "Microwave mediated organic reaction: a convenient approach for rapid and efficient synthesis of biologically active substituted 1,3-dihydro-2H-indol-2-one derivatives," J. Panda et al. synthesized some Schiff base of Isatin derivatives by conventional and microwave irradiation method. With the help of microwave synthesis, the yield of product was increased from $53 \%$ up to $84 \%$ as compared to conventional method. By microwave irradiation, the reactions were completed within 4-8 minutes, and the products were obtained in good to high yields, which reduced the time, waste, and formation of byproduct. Compounds substituted with electron withdrawing group in Isatin residue showed promising anthelmintic activities, whereas compounds containing electron donating group such as compound M8 exhibited poor activity.

In "Inclusion phenomena between the $\beta$-cyclodextrin chiral selector and $\operatorname{Trp}-D, L$, and its use on the assembly of solid membranes," H. Meng et al. prepared mono-6-deoxy-6(3-methylimidazolium)- $\beta$-cyclodextrin tosylate to improve the application of $\beta$-cyclodextrin for chiral separation. The separation factor between $\beta$-CD and $\beta$-CD-IL with D, L-Trp was studied by the saturated solution method and revealed that the chiral ionic liquid had a higher separation factor because of its high solubility. The selectivity was reduced as 
the $\beta$-CD-IL content within the membranes increased, while the sorption selectivity increased.

In their paper "Directed self-assembly of block copolymer for bit patterned media with areal density beyond 1 teradot/inch ${ }^{2}$," X. M. Yang et al. fabricated the high density master template using DSA approach through density multiplication. To fabricate a servo- and data-integrated BPM template, a 2-D prepattern can be used for the data zone, whereas a 1-D trench prepattern is used for the servo zone. Several challenging key BPM processes have been demonstrated, including template fabrication, imprint process, and magnetic dot formation. The bit-patterned magnetic media at $1.5 \mathrm{Td} /$ inch $^{2}$ have been successfully demonstrated.

In their paper "Patterned honeycomb structural films with fluorescent and hydrophobic properties," L. Heng et al. fabricated the patterned porous honeycomb-like film with the aggregation-induced emission (AIE) property by breath figure method. Characterization of contact angles indicated that the patterned porous honeycomb structure can improve the hydrophobicity of the film, while smooth films have not shown this function. Furthermore, characterization of the fluorescence property indicates that the patterned honeycomb structure films are highly emissive.

In "Sub-15 mn silicon lines fabrication via PS-b-PDMS

block copolymer lithography," S. Rasappa et al. described the fabrication of nanodimensioned silicon structures on silicon wafers from thin films of a poly (styrene)-block-poly (dimethylsiloxane) (PS-b-PDMS) block copolymer (BCP) precursor self-assembling into cylindrical morphology in the bulk. The structure alignment of the PS-b-PDMS (33 k$17 \mathrm{k}$ ) was conditioned by applying solvent and solvothermal annealing techniques. BCP nanopatterns formed after the annealing process have been confirmed by SEM after removal of upper PDMS wetting layer by plasma etching, and it revealed the formation of silicon nanostructures, notably of less than $15 \mathrm{~nm}$ dimensions.

\section{Acknowledgments}

We would like to express our sincere appreciation to all the authors for their contribution. Moreover, thanks are extended to all reviewers for their time and for enhancing the quality of these papers.

Amir Kajbafvala

Hamed Bahmanpour Mohammad H. Maneshian Minghang Li 

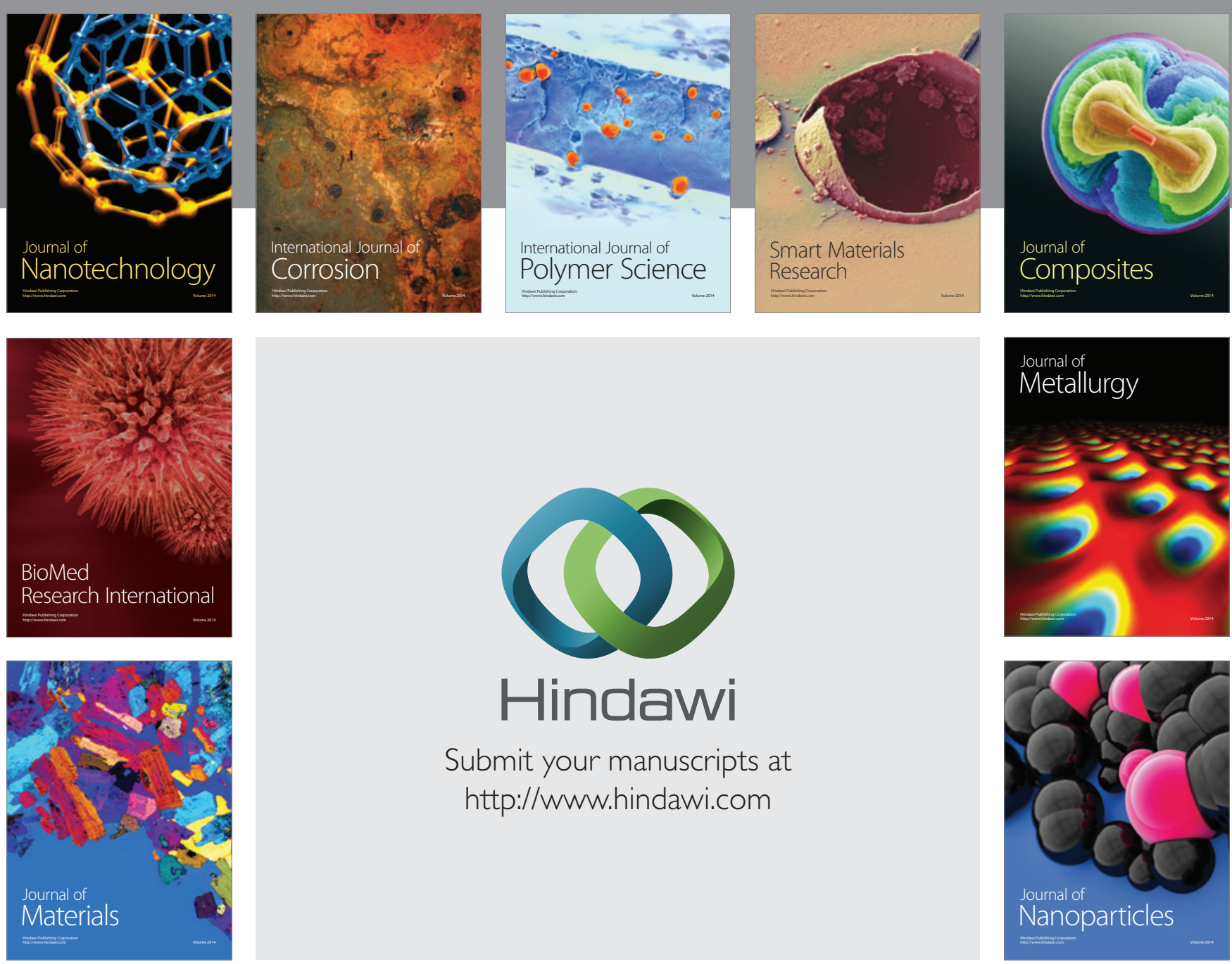

Submit your manuscripts at http://www.hindawi.com
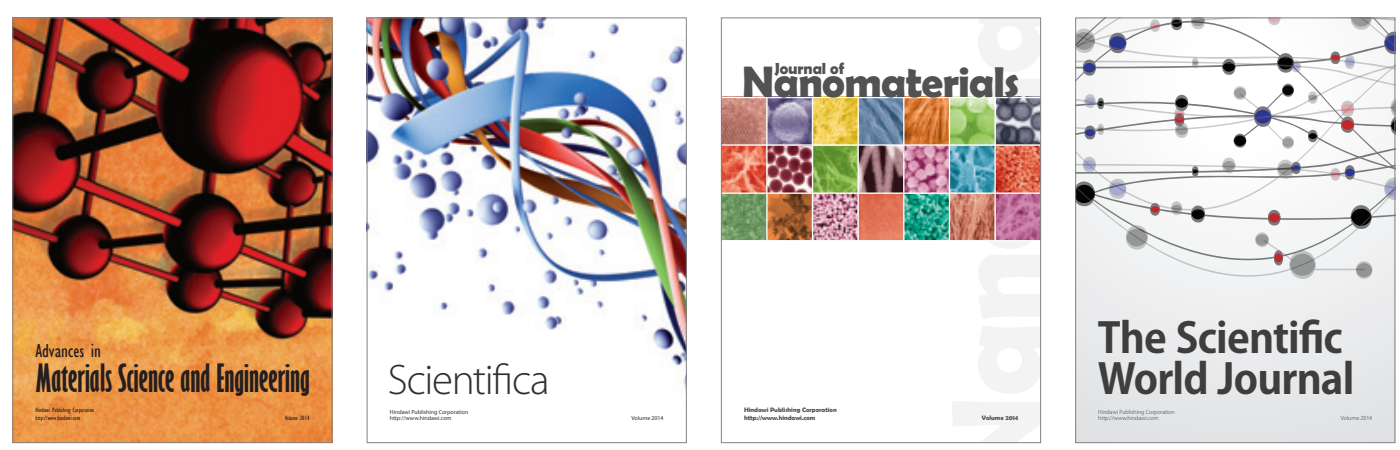

\section{The Scientific World Journal}
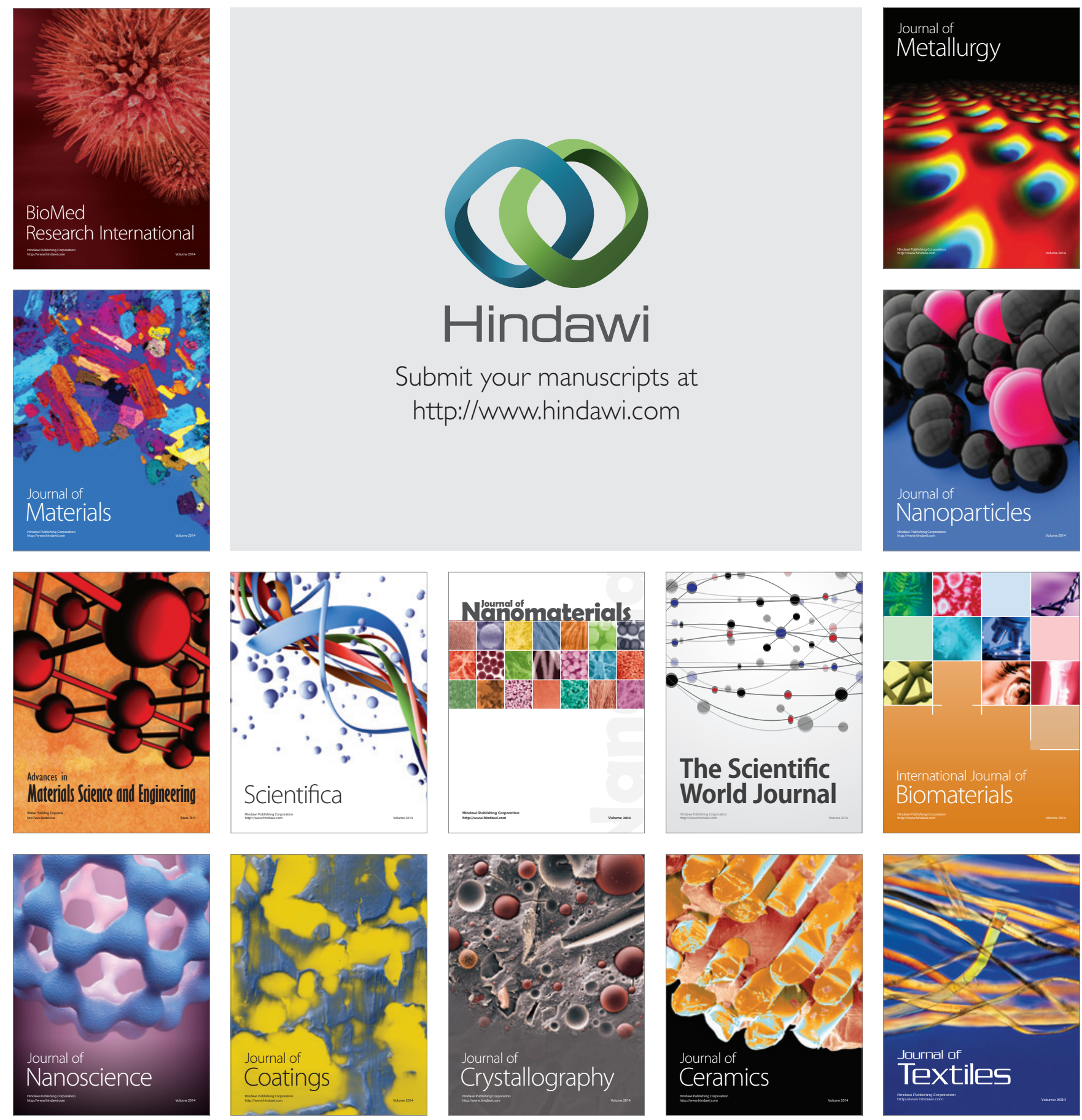